\title{
Dynamic Model Identification for 6-DOF Industrial Robots
}

\author{
Li Ding, ${ }^{1}$ Hongtao Wu, ${ }^{1}$ Yu Yao, ${ }^{2}$ and Yuxuan Yang' \\ ${ }^{1}$ College of Mechanical and Electrical Engineering, Nanjing University of Aeronautics and Astronautics, No. 29, Yudao Street, \\ Nanjing 210016, China \\ ${ }^{2}$ College of Aerospace Engineering, Nanjing University of Aeronautics and Astronautics, No. 29, Yudao Street, Nanjing 210016, China
}

Correspondence should be addressed to Hongtao Wu; mehtwu@126.com

Received 25 August 2015; Accepted 12 October 2015

Academic Editor: Keigo Watanabe

Copyright (C) 2015 Li Ding et al. This is an open access article distributed under the Creative Commons Attribution License, which permits unrestricted use, distribution, and reproduction in any medium, provided the original work is properly cited.

A complete and systematic procedure for the dynamical parameters identification of industrial robot manipulator is presented. The system model of robot including joint friction model is linear with respect to the dynamical parameters. Identification experiments are carried out for a 6-degree-of-freedom (DOF) ER-16 robot. Relevant data is sampled while the robot is tracking optimal trajectories that excite the system. The artificial bee colony algorithm is introduced to estimate the unknown parameters. And we validate the dynamical model according to torque prediction accuracy. All the results are presented to demonstrate the efficiency of our proposed identification algorithm and the accuracy of the identified robot model.

\section{Introduction}

In recent years, industrial robots have been greatly used as orienting devices in industry, especially in the shipbuilding, automotive, and aerospace manufacturing industries $[1,2]$. Advanced control techniques for robots have become more and more affordable thanks to increasing power of computing resources and their dramatic cost reduction. However, the dynamical model of robot contains uncertainties in some parameters and many control methods are sensitive to their values especially in high speed operations. Hence, dynamical parameters identification approach has importance for developing model based controllers.

In terms of academic research, a standard robot identification procedure consists of dynamic modeling, excitation trajectory design, data collection, signal preprocess, parameter identification, and model validation [3]. The parameter identification has attracted considerable attention from numerous researchers. Atkeson et al. [4] proposed the least square method to realize the estimation of dynamical parameters. Grotjahn et al. [5] used the two-step approach to perform the identification of robot dynamics. Gautier and Poignet [6] obtained a dynamical model of SCARA robot from experimental data with weighted least squares method. Behzad et al. [7] applied fractional subspace method to identify a robot model in simulation field. Recently, some intelligence computation algorithms have been reported as a useful tool in robot model identification. A traditional genetic algorithm (GA) was proposed to identify the autonomous underwater robot in [8]. Liu et al. [9] introduced the improved genetic algorithm to obtain the space robot model. However, while dealing with complex and large-scale parameters identification problems, the GA algorithm would be stuck on local optimum.

Artificial bee colony algorithm $(\mathrm{ABC})$ was first proposed by Karaboga in 2005 [10] and successfully applied to parameters identification of aerial robot [11]. The ABC algorithm has been proved to possess a better performance in function optimization problems, compared with differential evolution algorithm (DE), particle swarm optimization algorithm (PSO), and GA algorithm [12]. As we know, usual optimization algorithms conduct only one search operation in one iteration, but $\mathrm{ABC}$ algorithm can conduct both local search and global search in each iteration, and as a result the probability of finding the optimal parameters is significantly increased, which efficiently avoids local optimum to a large extent. In this paper, the $\mathrm{ABC}$ algorithm was introduced to conquer the parameters identification problem of the industrial robots. The identification experiment was implemented on 6-DOF ER-16 robot manipulator. 
The outline of this paper is organized as follows. Firstly, the linear robot dynamical model is given in Section 2. Then, Section 3 presents the identification process of the linear model based on the ABC algorithm, where excitation design, data collection, and signal preprocess are described. Later on, the experimental platform, identified results, and model validation are presented in Section 4. Finally the main conclusions are given in Section 5.

\section{Dynamic Modeling}

Since the $n$-DOF industrial robot is represented by a kinematic chain of rigid bodies, the exhaustive description for its motion can be found in [13]. The dynamic model of industrial robot is derived by the Newton-Euler or Lagrangian method:

$$
\boldsymbol{\tau}_{\mathrm{dyn}}=\mathbf{M}(q) \ddot{q}+\mathbf{C}(q, \dot{q})+\mathbf{G}(q)
$$

where $\boldsymbol{\tau}_{\text {dyn }}$ is the $n$-vector of actuator torques as well as the joint positions $q$, velocities $\dot{q}$, and accelerations $\ddot{q} . \mathbf{M}(q)$ is the $n \times n$ inertia matrix, $\mathbf{C}(q, \dot{q})$ denotes the $n$-vector including Coriolis and centrifugal forces, and $\mathbf{G}(q)$ is the $n$-vector of gravity.

According to the modified Newton-Euler parameters [14] or the barycentric parameters [15], (1) can be rewritten as a linear form:

$$
\boldsymbol{\tau}_{\mathrm{dyn}}=\boldsymbol{\Phi}_{\mathrm{dyn}}(q, \dot{q}, \ddot{q}) \boldsymbol{\theta}_{\mathrm{dyn}}
$$

where $\Phi_{\text {dyn }}$ denotes the $n \times 10 n$ observation or identification matrix, which depends only on the motion data. $\boldsymbol{\theta}_{\text {dyn }}$ is the barycentric parameter vector. This property considerably simplifies the parameters identification.

Dynamic model of robots also contains the torques caused by joint frictions and inertias of actuator rotors apart from the effects of dynamic parameters in (2). The inertias of actuator rotors are generally provided by producers, and corresponding torques should be compensated for the dynamic equations. In fact, joint friction is a complex nonlinear model, especially during motion reversal. In order to simplify the model, the friction model consisting of only Coulomb and viscous friction [16] is given by

$$
\boldsymbol{\tau}_{\text {fric }}=f_{c} \operatorname{sign}(\dot{q})+f_{v} \dot{q},
$$

where $\boldsymbol{\tau}_{\text {fric }}$ is the friction torques and $f_{c}, f_{v}$, respectively, mean the Coulomb and viscous friction parameters.

The integrated dynamic model of robots can be written as

$$
\boldsymbol{\tau}_{s}=\Phi_{s}(q, \dot{q}, \ddot{q}) \boldsymbol{\theta}_{s}
$$

where $\boldsymbol{\tau}_{s}$ is actuator torques including $\boldsymbol{\tau}_{\text {dyn }}$ and $\boldsymbol{\tau}_{\text {fric }} . \Phi_{s}$ is the $n \times 12 n$ observation matrix, and $\boldsymbol{\theta}_{s}$ is $12 n$-vector of unknown dynamic parameters. In addition, the dynamic parameters of link $i$ are governed by the form:

$$
\begin{gathered}
\boldsymbol{\theta}_{s}^{i}=\left[I_{x x i}, I_{x y i}, I_{x z i}, I_{y y i}, I_{y z i}, I_{z z i}, m_{i} r_{x i}, m_{i} r_{y i}, m_{i} r_{z i}, m_{i}, f_{c i},\right. \\
\left.f_{v i}\right]^{T},
\end{gathered}
$$

where $I_{\zeta \zeta i}(\zeta=x, y, z)$ is the inertial tensor of link $i$. Similarly, $m_{i} r_{\zeta i}$ denotes the first-order mass moment and $m_{i}$ is the mass of link $i$.

In general, the observation matrix $\Phi_{s}$ in (4) is not a full rank; that is, not all dynamic parameters have an influence on the dynamic model. In order to obtain a set of minimum parameters, a case-by-case analysis method is adopted [17]. Consequently, the dynamic model based on the basic dynamic parameters can be rewritten as

$$
\boldsymbol{\tau}=\boldsymbol{\Phi}(q, \dot{q}, \ddot{q}) \boldsymbol{\theta},
$$

where $\boldsymbol{\Phi}$ is the $n \times(p+2 n)$ observation matrix. $\boldsymbol{\theta}$ is $(p+2 n)$ vector of dynamic parameters, including the basic parameters and the friction parameters. $p$ denotes the number of the minimum dynamical parameters. $2 n$ denotes the number of the friction parameters.

\section{Parameters Identification Procedures}

3.1. Basic Principles of Identification Algorithm. In order to introduce the search mechanism of ABC algorithm, we should define three essential components: employed bees, unemployed bees, and food source [11]. And the unemployed bees are divided into following bees and scout bees. The population of the colony bees is $N_{s}$, the number of employed bees is $N_{e}$, and the number of unemployed bees is $N_{u}$, which satisfies the relation $N_{s}=2 N_{e}=2 N_{u}$. We also define $D$ as the dimension of solution vector, that is, the number of the unknown parameters. ABC algorithm treats each unknown parameter as a food source. The detailed procedure of executing the proposed algorithm is described as follows.

Step 1. Randomly initialize a set of possible solutions $\left(x_{1}, \ldots, x_{N_{s}}\right)$, and the particular solution $x_{i}$ can be governed by

$$
x_{i}^{j}=x_{\min }^{j}+\operatorname{rand}(0,1)\left(x_{\max }^{j}-x_{\min }^{j}\right),
$$

where $j \in\{1, \ldots, D\}$ denotes the $j$ th dimension of the solution vector. $x_{\min }^{j}$ and $x_{\max }^{j}$ mean the lower and upper bounds, respectively.

Step 2. Apply a specific function to calculate the fitness of the solution $x_{i}$ according to the following equations and select the top $N_{e}$ best solutions as the number of the employed bees:

$$
\begin{aligned}
\text { fit }_{i} & =\frac{1}{\left(1+F_{i}\right)} \\
F_{i} & =\frac{1}{N} \sum_{i=1}^{N}\left(\delta_{1}\left\|\tau_{1 i}-\tau_{p 1 i}\right\|+\delta_{2}\left\|\tau_{2 i}-\tau_{p 2 i}\right\|\right. \\
& \left.+\delta_{3}\left\|\tau_{3 i}-\tau_{p 3 i}\right\|\right)
\end{aligned}
$$

where fit ${ }_{i}$ is the fitness function, $F_{i}$ is the objective function, $N$ is the data length, and $\tau_{\xi i}(\xi=1,2,3)$ is the vector of the actual torques data from the first three joints. Similarly, $\tau_{p \xi i}(\xi=1,2,3)$ is the vector of the predicted data from the identified model. $\delta_{\xi}(\xi=1,2,3)$ is a weight coefficient between 0 and 1 . 
Step 3. Each employed bee searches new solution in the neighborhood of the current position vector in the $n$th iteration as follows:

$$
v_{i}^{j}=x_{i}^{j}+\lambda_{i}^{j}\left(x_{i}^{j}-x_{k}^{j}\right),
$$

where $k \in\{1, \ldots, D\}, k \neq i$, both $k$ and $j$ are randomly generated, and $\lambda_{i}^{j}$ is a random parameter in the range from -1 to 1 . Next, we apply the greedy selection equation (11) to choose the better solution between $v_{i}^{j}$ and $x_{i}^{j}$ into the next generation:

$$
x_{i}^{j}= \begin{cases}v_{i}^{j}, & \text { fit }\left(v_{i}^{j}\right)>\text { fit }\left(x_{i}^{j}\right), \\ x_{i}^{j}, & \text { fit }\left(v_{i}^{j}\right) \leq \text { fit }\left(x_{i}^{j}\right) .\end{cases}
$$

Step 4. Each following bee selects an employed bee to trace according to the parameter of probability value. The formula of the probability method is described as

$$
p_{i}=\frac{\text { fit }_{i}}{\sum_{i=1}^{N e} \mathrm{fit}_{i}} .
$$

Step 5. The following bee searches in the neighborhood of the selected employed bee's position to find new solutions. Update the current solution according to their fitness.

Step 6. If the search time trial is larger than the predetermined threshold limit and the optimal value cannot be improved, then the location vector can be reinitialized randomly by scout bees according to the following equation:

$$
\begin{aligned}
& x_{i}(n+1) \\
& = \begin{cases}x_{\min }+\operatorname{rand}(0,1)\left(x_{\max }-x_{\min }\right), & \text { trial }>\text { limit, } \\
x_{i}(n), & \text { trial } \leq \text { limit. }\end{cases}
\end{aligned}
$$

Step 7. Output the best solution parameters achieved at the present time, and go back to Step 3 until termination criterion $T_{\max }$ is met.

The detailed procedure of $\mathrm{ABC}$ algorithm for parameters identification can be also depicted in Figure 1.

3.2. Excitation Trajectory. When designing an identification experiment for the robot, it is necessary to design proper excitation trajectories to ensure the accuracy of estimation in presence of disturbances [18]. In this work, a finite Fourier series is adopted as excitation trajectories, that is, a finite sum of harmonic sine and cosine functions. The trajectories for joint $i$ of a robot are designed as

$$
q_{i}(t)=q_{i, o}+\sum_{k=1}^{N} a_{i, k} \sin \left(k \omega_{f} t\right)+\sum_{k=1}^{N} b_{i, k} \cos \left(k \omega_{f} t\right),
$$

where $q_{i, o}$ is the offset term and $\omega_{f}$ is the fundamental pulsation of the Fourier series. This Fourier series specifies a periodic function with period $T_{f}=2 \pi / \omega_{f}$. Each Fourier

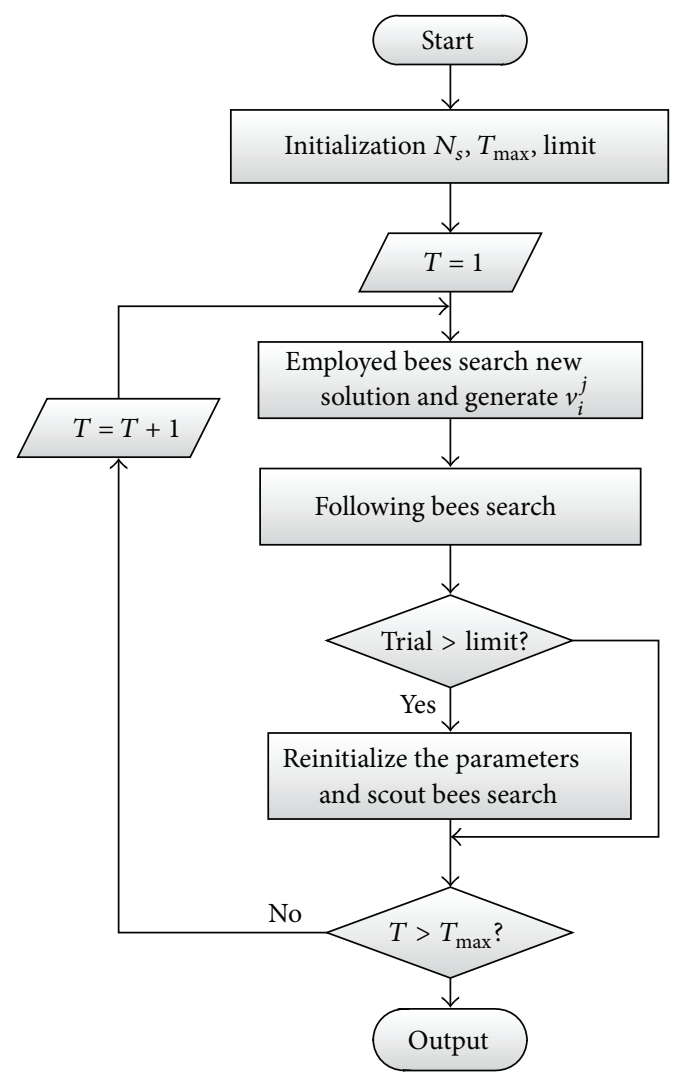

FIGURE 1: Sketch of the identification algorithm.

series contains $2 N+1$ parameters, and $a_{i, k}, b_{i, k}$ are the amplitudes of the sine and cosine functions.

The noise immunity and convergence rate of an identification experiment depend directly upon the constraints of the excitation trajectories. It is important to emphasize that the configurations for which measurements are taken must correspond to a well-conditioned reduced observation matrix since the constraints represent some limits for input/output. In the literature, the constraints of the excitation trajectories can be described as

$$
\begin{gathered}
\text { mincond }(\Phi) \\
q_{\min } \leq q(\beta) \leq q_{\max }, \\
|\dot{q}(\beta)| \leq \dot{q}_{\max } \\
|\ddot{q}(\beta)| \leq \ddot{q}_{\max } \\
w(q(\beta)) \subset W_{o} \\
\tau_{\min } \leq \Phi(q(\beta), \dot{q}(\beta), \ddot{q}(\beta)) \theta \leq \tau_{\max },
\end{gathered}
$$

where $q_{\min }$ and $q_{\max }$ are the lower and upper of the joint positions, $\dot{q}_{\max }$ and $\ddot{q}_{\max }$ are the upper of velocities and accelerations, $\beta$ is optimal trajectory parameters, $W_{o}$ is the available workspace of robot, and $\tau_{\max }$ is the maximum joint torque. 
3.3. Preprocessing of Measured Data. The measured torques are obtained through collecting the data of motor current, which is described as follows:

$$
\tau=K I,
$$

where $I$ is motor current. $K$ is just coefficient.

Since there are measurement noises in experiments, it is necessary to preprocess the collection data before identification. In order to remove outliers and attenuate the effect of interference signal, a five-spot triple smoothing method is adopted to smooth the raw data according to the following equations:

$$
\begin{aligned}
& \bar{y}_{1}=\frac{1}{70}\left[69 y_{1}+4\left(y_{2}+y_{4}\right)-6 y_{3}-y_{5}\right], \\
& \bar{y}_{2}=\frac{1}{35}\left[2\left(y_{1}+y_{5}\right)+27 y_{2}+12 y_{3}-8 y_{4}\right], \\
& \bar{y}_{i}=\frac{1}{35}\left[-3\left(y_{i-2}+y_{i+2}\right)+12\left(y_{i-1}+y_{i+1}\right)+17 y_{i}\right], \\
& \bar{y}_{m-1} \\
& \quad=\frac{1}{35}\left[2\left(y_{m-4}+y_{m}\right)-8 y_{m-3}+12 y_{m-2}+27 y_{m-1}\right], \\
& \bar{y}_{m} \\
& \quad=\frac{1}{70}\left[-y_{m-4}+4\left(y_{m-3}+y_{m-1}\right)-6 y_{m-2}+69 y_{m}\right],
\end{aligned}
$$

where $i=3, \ldots, m-2, \mathbf{Y}=\left[y_{1}, y_{2}, \ldots, y_{m}\right]$ is the measured raw data, and $\overline{\mathbf{Y}}=\left[\bar{y}_{1}, \ldots, \bar{y}_{m}\right]^{T}$ is the data for identification after preprocessing. The more the number of using (17) is, the smoother the curves will be. It should be noted that excessively using (17) to smooth the raw data can lead to the error of the parameters identification increasing.

In addition, the velocities and accelerations of joints cannot be measured directly. However, these pieces of information are usually obtained by joint positions, and numerical differentiation for joint positions can amplify the measurement noise and decrease accuracy of the velocities and accelerations. An analytical approach is adopted to overcome the aforementioned difficulty, which was proposed in [19] and used successfully in [20]. The average joint positions are approximated as finite Fourier series through the linear least square technique and the joint velocities and accelerations can be estimated by the derivatives of the obtained finite Fourier series.

\section{Experiment Results}

4.1. Parameters Identification. An experiment is conducted to test the proposed identification algorithm. The ER-16, shown in Figure 2, is a 6-DOF industrial robot manipulator without payload. And the link frame of the robot is depicted in Figure 3. The geometric parameters of the ER-16 robot are given in Table 1. Only the first joints are considered here. A fundamental pulsation of $0.05 \mathrm{~Hz}$ is selected for the excitation trajectories, resulting in a period of $25 \mathrm{~s}$. As shown in Figure 4, the commanded trajectories are five-term

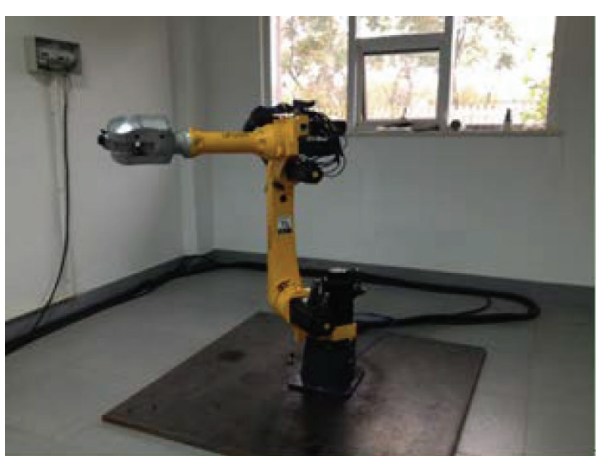

FIGURE 2: ER-16 6-DOF robot.

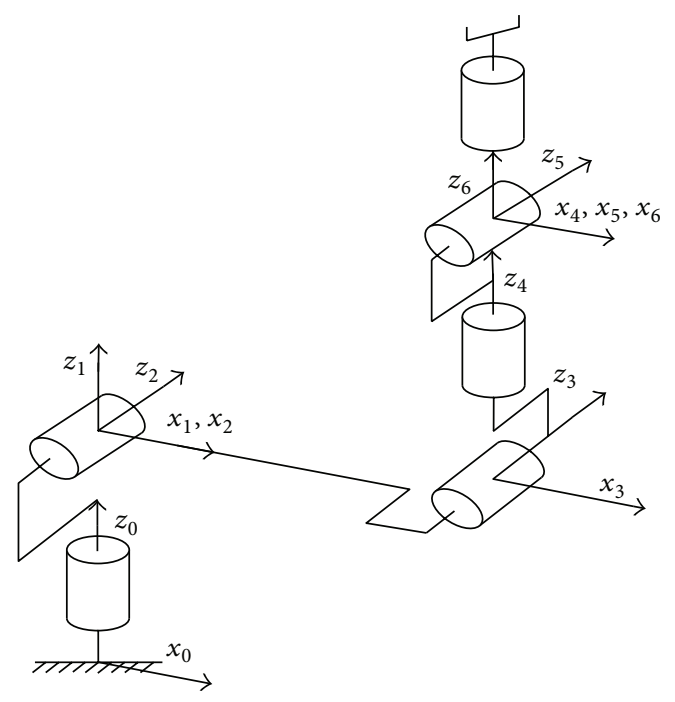

FIGURE 3: The link frame of ER-16 robot.

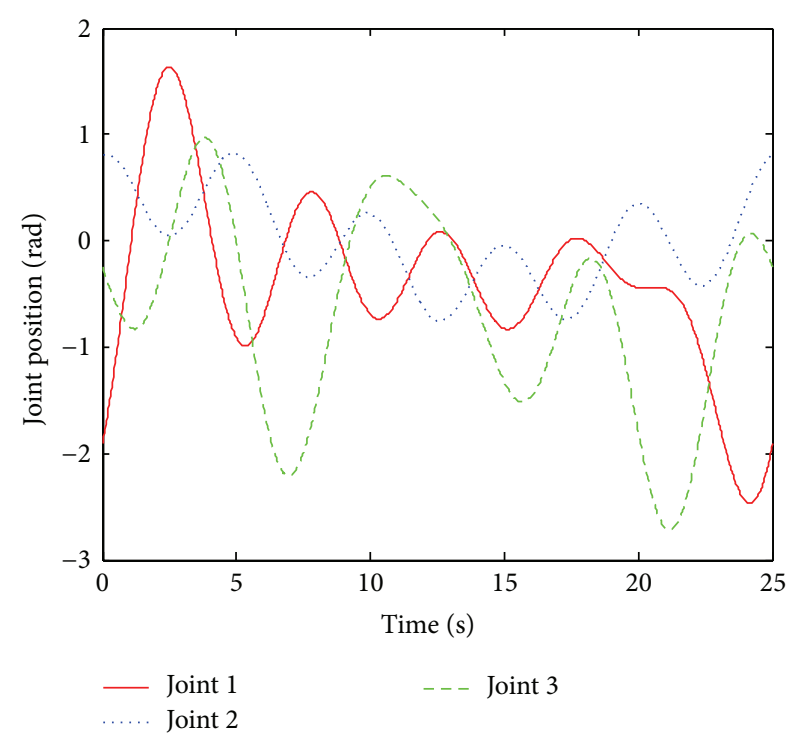

FIGURE 4: Optimized robot excitation trajectories. 
TABLE 1: DH parameters of ER-16 robot.

\begin{tabular}{lcccc}
\hline Link $i$ & $\alpha_{i-1}(\mathrm{rad})$ & $a_{i-1}(\mathrm{~m})$ & $d_{i}(\mathrm{~m})$ & $\theta_{i}(\mathrm{rad})$ \\
\hline 1 & $\pi$ & 0 & 0 & $\theta_{1}$ \\
2 & $\pi / 2$ & 0.16 & 0 & $\theta_{2}-\pi / 2$ \\
3 & 0 & 0.68 & 0 & $\theta_{3}$ \\
4 & $\pi / 2$ & 0.13 & -0.75 & $\theta_{4}$ \\
5 & $-\pi / 2$ & 0 & 0 & $\theta_{5}$ \\
6 & $\pi / 2$ & 0 & 0 & $\theta_{6}$ \\
\hline
\end{tabular}

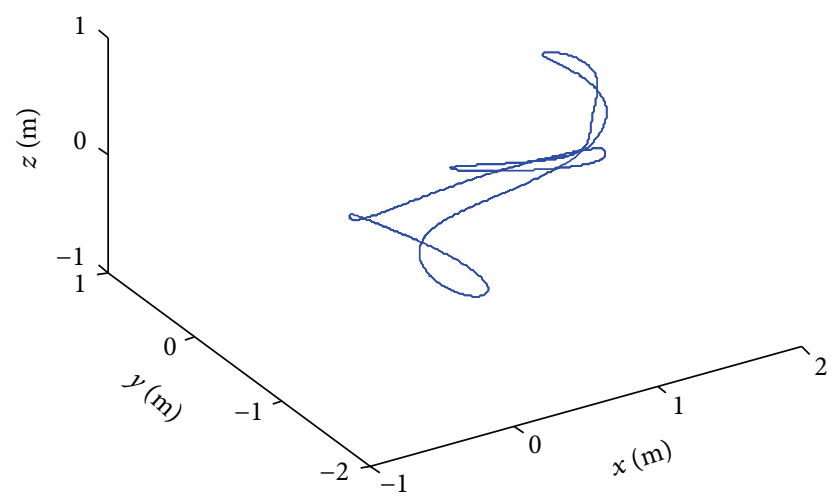

FIGURE 5: 3D visualization of the optimized trajectory.

Fourier series, involving 11 optimal trajectory parameters for each joint which are listed in the Appendices, and a $0.25 \mathrm{~Hz}$ bandwidth. The 3D visualization of this optimized trajectory in the workspace of the robot is shown in Figure 5. The total measured time is $25 \mathrm{~s}$, corresponding to 1 period of the excitation trajectory. The data is sampled with $1 \mathrm{kHz}$.

Identification procedures are carried out with $\mathrm{ABC}$ algorithm in Matlab 2013b programming environment on an Intel Core i7-3770 PC running Windows 7. No commercial tools are used. According to [12], the performance of $\mathrm{ABC}$ algorithm is relative to the population size of colony bees. As the population size increases, the algorithm produces better results. However, after a sufficient value for colony size, any increment in the value does not improve the performance of $\mathrm{ABC}$ algorithm. And the control parameter limit is based on location vector reinitialized frequency. As the value of limit approaches infinity, the total number of location vectors reinitialized goes to zero. After many trials, in this paper, we set the parameters of $\mathrm{ABC}$ algorithm as follows: $N_{s}=$ 30 , limit $=15$, and $T_{\max }=50$. It should be noted that when the predetermined iterations exceed 50, the $A B C$ algorithm converged and the objective value could not be obviously improved. And the search scope of the unknown parameters is also listed in the Appendices. The objective value of optimization process for the parameters is shown in Figure 6. The result shows that the convergence speed of our algorithm is fast and the final objective value which is calculated by (9) is 0.3182 .

The robot dynamic model for the first joints contains 21 parameters, 15 base parameters, and 6 friction parameters.

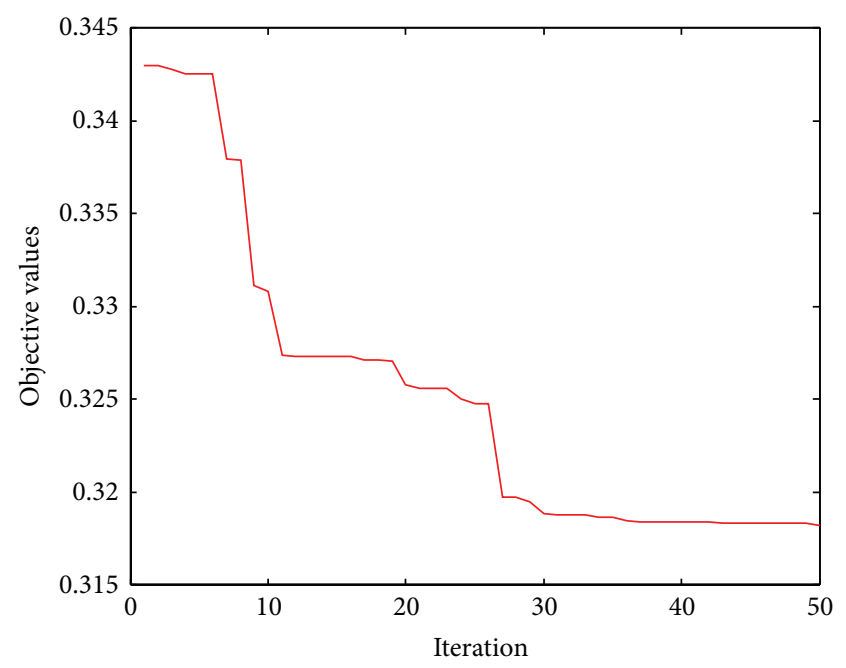

FIGURE 6: Evolutionary curves of identification algorithm.

TABLE 2: Identification dynamic parameters.

\begin{tabular}{lc}
\hline Parameter & Value \\
\hline$I_{z z 1}\left(\mathrm{~kg} \cdot \mathrm{m}^{2}\right)$ & 50.4438 \\
$I_{x x 2}\left(\mathrm{~kg} \cdot \mathrm{m}^{2}\right)$ & 37.7503 \\
$I_{x y 2}\left(\mathrm{~kg} \cdot \mathrm{m}^{2}\right)$ & 16.1693 \\
$I_{x z 2}\left(\mathrm{~kg} \cdot \mathrm{m}^{2}\right)$ & -23.0075 \\
$I_{y z 2}\left(\mathrm{~kg} \cdot \mathrm{m}^{2}\right)$ & 3.2047 \\
$I_{z z 2}\left(\mathrm{~kg} \cdot \mathrm{m}^{2}\right)$ & 1.5512 \\
$m_{2} r_{x 2}(\mathrm{~kg} \cdot \mathrm{m})$ & 5.9390 \\
$m_{2} r_{y 2}(\mathrm{~kg} \cdot \mathrm{m})$ & 46.8898 \\
$I_{x x 3}\left(\mathrm{~kg} \cdot \mathrm{m}^{2}\right)$ & 30.9319 \\
$I_{x y 3}\left(\mathrm{~kg} \cdot \mathrm{m}^{2}\right)$ & -0.7515 \\
$I_{y z 3}\left(\mathrm{~kg} \cdot \mathrm{m}^{2}\right)$ & 63.2997 \\
$I_{z z 3}\left(\mathrm{~kg} \cdot \mathrm{m}^{2}\right)$ & -0.4069 \\
$m_{3} r_{x 3}(\mathrm{~kg} \cdot \mathrm{m})$ & 2.3608 \\
$m_{3} r_{y 3}(\mathrm{~kg} \cdot \mathrm{m})$ & 1.4471 \\
$f_{c 1}(\mathrm{~N} \cdot \mathrm{m})$ & -0.0448 \\
$f_{v 1}(\mathrm{Nm} \cdot \mathrm{s} / \mathrm{rad})$ & 0.1144 \\
$f_{c 2}(\mathrm{~N} \cdot \mathrm{m})$ & 11.0195 \\
$f_{v 2}(\mathrm{Nm} \cdot \mathrm{s} / \mathrm{rad})$ & 5.3853 \\
$f_{c 3}(\mathrm{~N} \cdot \mathrm{m})$ & 10.9173 \\
$f_{v 3}(\mathrm{Nm} \cdot \mathrm{s} / \mathrm{rad})$ & 27.3475 \\
$I_{x z 3}\left(\mathrm{~kg} \cdot \mathrm{m}^{2}\right)$ & 2.3092 \\
\hline &
\end{tabular}

The parameters identified by our proposed algorithm are listed in Table 2. It should be noted that the values of the dynamic parameters of the first three joints are much bigger than those of the other three joints. It is reasonable to ignore the effect of the torques caused by the 4,5 , and 6 joints. Figure 7 compares the measured torques for the excitation trajectories with the predicted torques based on the identified dynamical model. Although the results show that the predicted error is slightly big during velocity reversal, the predicted torques have the same trend as the measured 


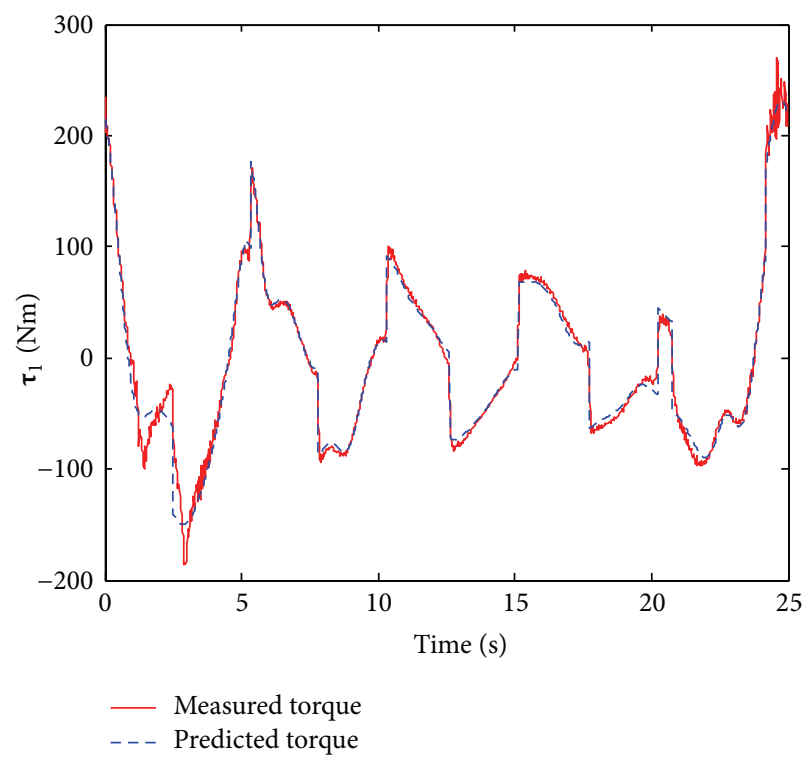

(a) Joint 1

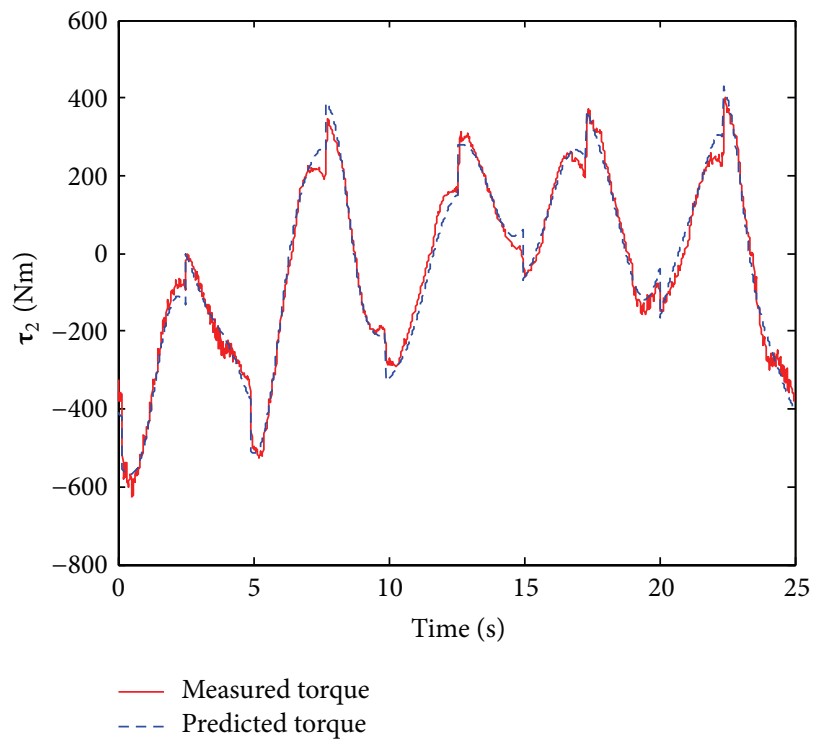

(b) Joint 2

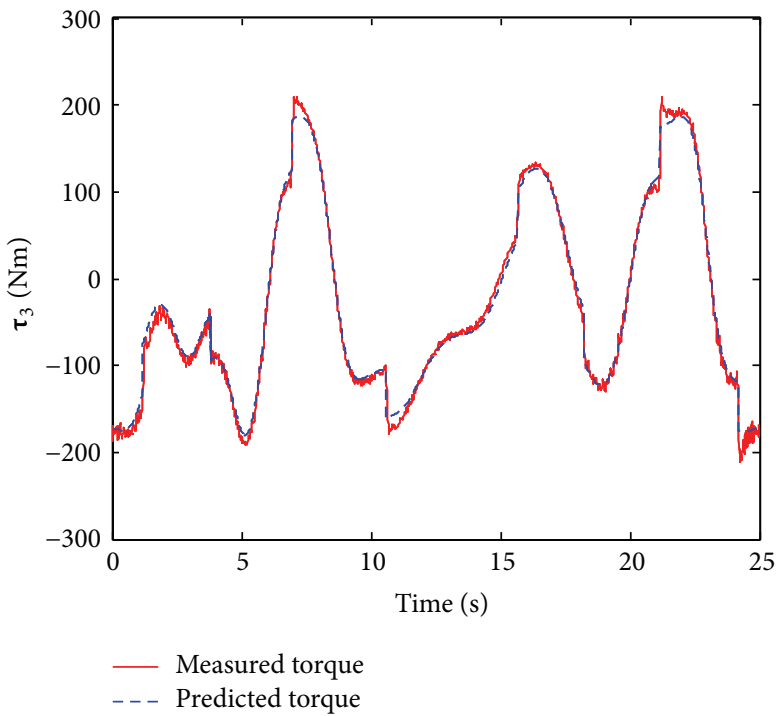

(c) Joint 3

FIGURE 7: Comparison of the measured torques and predicted torques.

torques. It indicates that $\mathrm{ABC}$ algorithm has a strong ability to find the optimal parameters.

To verify the precision of the identified model by $\mathrm{ABC}$ algorithm, the correlation coefficient between the measured torques $\boldsymbol{\tau}_{i}$ and predicted torques $\boldsymbol{\tau}_{p i}$, defined as the normalized cross-covariance function, is applied to estimate how well the identified model can reproduce the measured torques, and the function is defined as

$$
\rho=\frac{\sum_{i=1}^{N}\left(\boldsymbol{\tau}_{i}-\overline{\boldsymbol{\tau}}\right)\left(\boldsymbol{\tau}_{m i}-\overline{\boldsymbol{\tau}}_{m}\right)}{\sqrt{\sum_{i=1}^{N}\left(\boldsymbol{\tau}_{i}-\overline{\boldsymbol{\tau}}\right)^{2} \sum_{i=1}^{N}\left(\boldsymbol{\tau}_{m i}-\overline{\boldsymbol{\tau}}_{m}\right)^{2}}},
$$

where $\overline{\boldsymbol{\tau}}=(1 / N) \sum_{i=1}^{N} \boldsymbol{\tau}_{i}$ and $\overline{\boldsymbol{\tau}}_{m}=(1 / N) \sum_{i=1}^{N} \overline{\boldsymbol{\tau}}_{m i}$. The closer the correlation coefficient is to unity, the better the identified model is. While the coefficient is close to zero, the identified model is poor. As a result, the correlation coefficients of the predicted first joints are $0.9533,0.9856$, and 0.9801 , respectively. It indicates that the identified parameters have satisfactory precision.

4.2. Model Validation. Since our aim for current stage is to investigate the validity of the model calculated by our proposed method, we focus on the validation test. Obviously, the appropriate validation test is to use the identified model in application and evaluate its success. As shown in Figure 8, three-term Fourier series are chosen as the excitation trajectories the same as the aforementioned excitation trajectories. Corresponding optimal trajectory parameters for each joint are listed in the Appendices. And the comparison of the measured and predicted torques is shown in Figure 9. It 


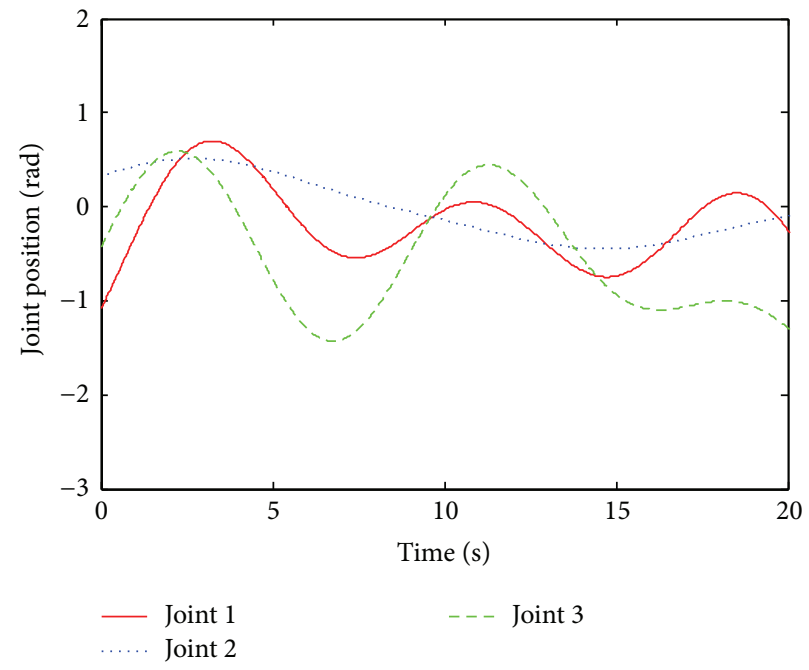

FIgURE 8: Robot model validation trajectory.
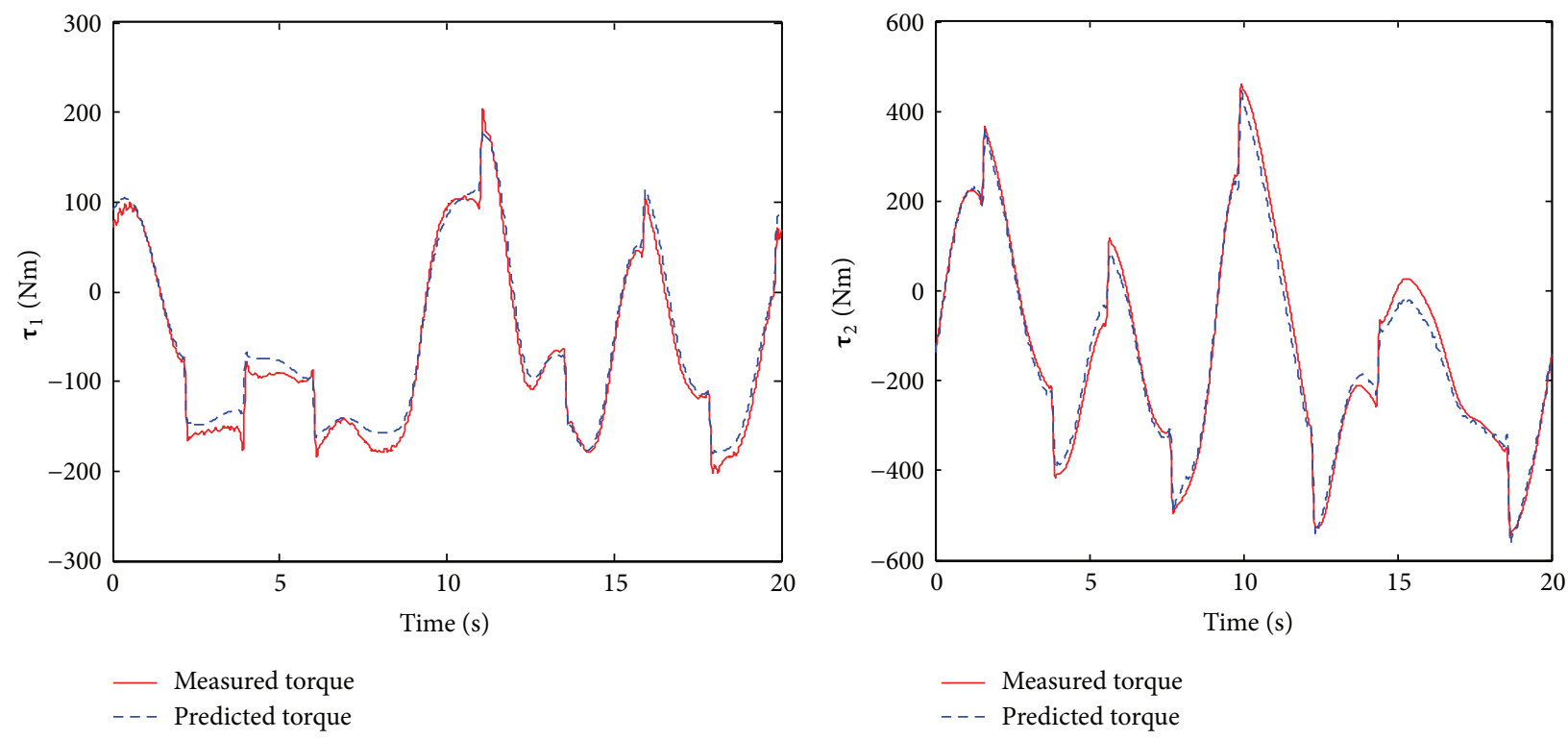

(a) Joint 1

(b) Joint 2

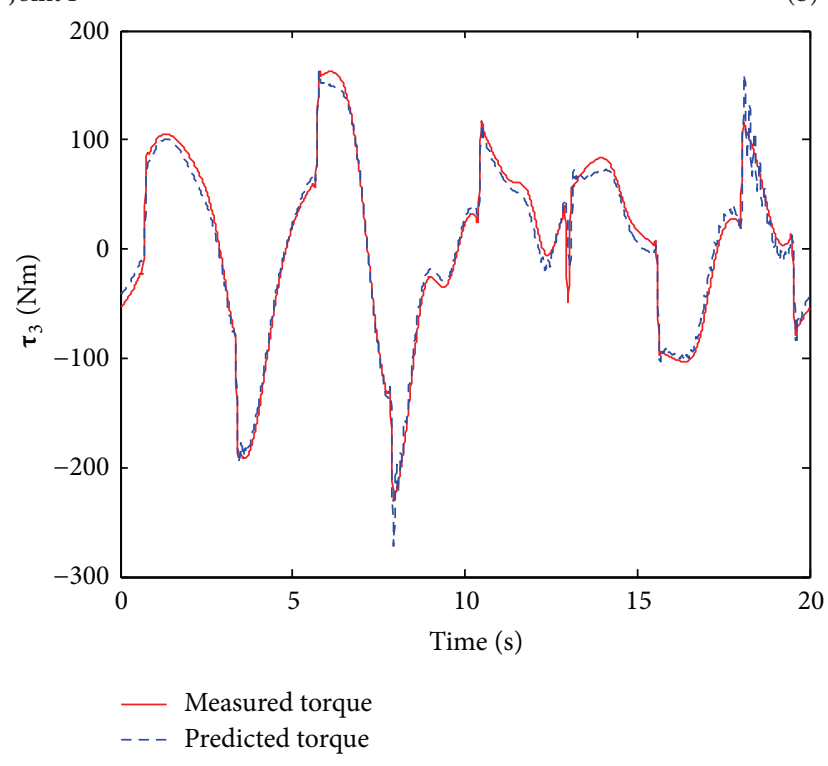

(c) Joint 3

FIGURE 9: Comparison of the measured torques and predicted torques. 
TABLE 3: Search scope of dynamical parameters.

\begin{tabular}{lc}
\hline Parameter & Scope \\
\hline$I_{z z 1}\left(\mathrm{~kg} \cdot \mathrm{m}^{2}\right)$ & {$[-60,60]$} \\
$I_{x x 2}\left(\mathrm{~kg} \cdot \mathrm{m}^{2}\right)$ & {$[-60,60]$} \\
$I_{x y 2}\left(\mathrm{~kg} \cdot \mathrm{m}^{2}\right)$ & {$[-30,30]$} \\
$I_{x z 2}\left(\mathrm{~kg} \cdot \mathrm{m}^{2}\right)$ & {$[-40,40]$} \\
$I_{y z 2}\left(\mathrm{~kg} \cdot \mathrm{m}^{2}\right)$ & {$[-10,10]$} \\
$I_{z z 2}\left(\mathrm{~kg} \cdot \mathrm{m}^{2}\right)$ & {$[-10,10]$} \\
$m_{2} r_{x 2}(\mathrm{~kg} \cdot \mathrm{m})$ & {$[-10,10]$} \\
$m_{2} r_{y 2}(\mathrm{~kg} \cdot \mathrm{m})$ & {$[-80,80]$} \\
$I_{x x 3}\left(\mathrm{~kg} \cdot \mathrm{m}^{2}\right)$ & {$[-60,60]$} \\
$I_{x y 3}\left(\mathrm{~kg} \cdot \mathrm{m}^{2}\right)$ & {$[-10,10]$} \\
$I_{y z 3}\left(\mathrm{~kg} \cdot \mathrm{m}^{2}\right)$ & {$[-80,80]$} \\
$I_{z z 3}\left(\mathrm{~kg} \cdot \mathrm{m}^{2}\right)$ & {$[-10,10]$} \\
$m_{3} r_{x 3}(\mathrm{~kg} \cdot \mathrm{m})$ & {$[-10,10]$} \\
$m_{3} r_{y 3}(\mathrm{~kg} \cdot \mathrm{m})$ & {$[-10,10]$} \\
$f_{c 1}(\mathrm{~N} \cdot \mathrm{m})$ & {$[-10,10]$} \\
$f_{v 1}(\mathrm{Nm} \cdot \mathrm{s} / \mathrm{rad})$ & {$[-10,10]$} \\
$f_{c 2}(\mathrm{~N} \cdot \mathrm{m})$ & {$[-10,10]$} \\
$f_{v 2}(\mathrm{Nm} \cdot \mathrm{s} / \mathrm{rad})$ & {$[-30,30]$} \\
$f_{c 3}(\mathrm{~N} \cdot \mathrm{m})$ & {$[-30,30]$} \\
$f_{v 3}(\mathrm{Nm} \cdot \mathrm{s} / \mathrm{rad})$ & {$[-30,30]$} \\
$I_{x z 3}\left(\mathrm{~kg} \cdot \mathrm{m}{ }^{2}\right)$ & {$[-10,10]$} \\
\hline
\end{tabular}

indicates that the model we obtain is capable of accurately predicting the actuator torque data. In addition, the correlation coefficients of the predicted first joints are 0.9272 , 0.9534 , and 0.9606 according to (18). The validation test not only shows very good results but also demonstrates that our proposed identification method is reliable enough.

\section{Conclusion}

In this paper a systematic procedure for the dynamical parameters identification of a $6-\mathrm{DOF}$ industrial robot has been presented. We design optimal periodic excitation trajectories to integrate the identification experiment, data collection, and signal preprocess. All the unknown parameters are well identified by $\mathrm{ABC}$ algorithm. When comparing the measured torques and the predicting torques, we conclude that our proposed method can accurately estimate the robot dynamical parameters. Further, a model validation has been carried out to verify the validity of the identified model. The results of this paper are useful for researchers and manufactures of industrial robots.

\section{Appendices}

\section{A. Optimal Parameters of Excitation Trajectories}

The optimal trajectory parameters for the five-term Fourier series are listed as follows:

$$
\beta_{1}=\left[\begin{array}{ccccccccccc}
-0.3857 & -0.1911 & -0.2842 & -0.2289 & -0.2455 & -0.5705 & 0.3359 & 0.4129 & 0.5744 & 0.4842 & 0.0730 \\
0.0011 & 0.3597 & -0.0179 & -0.0117 & 0.0443 & 0.4355 & 0.2551 & 0.0386 & 0.0411 & 0.0057 & -0.0065 \\
-0.6641 & -0.1638 & 0.5347 & -0.1448 & 0.1029 & 0.0826 & 0.4426 & 0.3359 & 0.6126 & -0.6714 & -0.5792
\end{array}\right] .
$$

The optimal trajectory parameters for the three-term Fourier series are listed as follows:

$$
\beta_{2}=\left[\begin{array}{ccccccc}
0.3016 & 0.4006 & 0.6921 & -0.4210 & -0.0423 & 0.6066 & -0.9719 \\
-0.5026 & -0.0051 & 0.0492 & 0.8074 & 0.4578 & -0.2521 & 0.6288 \\
-0.1276 & -0.0624 & 0.3805 & 0.4006 & 0.3016 & 0.3531 & 0.1524
\end{array}\right]
$$

\section{B. Search Scope of Dynamical Parameters}

After many trials, the probable suboptimal or optimal search scope of the unknown parameters is listed in Table 3.

\section{Conflict of Interests}

The authors declare that there is no conflict of interests regarding the publication of this paper.

\section{Acknowledgments}

This work was partially supported by the National Natural Sciences Foundation of China (51375230) and the project of Science and Technology Support Plan of Jiangsu Province (BE2013003-1, BE2013010-2).

\section{References}

[1] J. A. Persson, X. Feng, D. Wappling, and J. Ölvander, "A framework for multidisciplinary optimization of a balancing 
mechanism for an industrial robot," Journal of Robotics, vol. 2015, Article ID 389769, 8 pages, 2015.

[2] A. Paoli and A. V. Razionale, "Large yacht hull measurement by integrating optical scanning with mechanical tracking-based methodologies," Robotics and Computer-Integrated Manufacturing, vol. 28, no. 5, pp. 592-601, 2012.

[3] J. Wu, J. Wang, and Z. You, "An overview of dynamic parameter identification of robots," Robotics and Computer-Integrated Manufacturing, vol. 26, no. 5, pp. 414-419, 2010.

[4] C. G. Atkeson, C. H. An, and J. M. Hollerbach, "Estimation of inertial parameters of manipulator loads and links," International Journal of Robotics Research, vol. 5, no. 3, pp. 101-119, 1986.

[5] M. Grotjahn, M. Daemi, and B. Heimann, "Friction and rigid body identification of robot dynamics," International Journal of Solids and Structures, vol. 38, no. 10, pp. 1889-1902, 2001.

[6] M. Gautier and P. Poignet, "Extended Kalman filtering and weighted least squares dynamic identification of robot," Control Engineering Practice, vol. 9, no. 12, pp. 1361-1372, 2001.

[7] H. Behzad, H. T. Shandiz, A. Noori, and T. Abrishami, "Robot identification using fractional subspace method," in Proceedings of the 2nd International Conference on Control, Instrumentation and Automation (ICCIA '11), pp. 1193-1199, Shiraz, Iran, December 2011.

[8] J. Xuan, J. G. Liu, and S. F. Zhu, "Identification method of hydrodynamic parameters of autonomous underwater vehicle based on genetic algorithm," Journal of Mechanical Engineering, vol. 46, no. 11, pp. 96-100, 2010.

[9] Y. Liu, G.-X. Li, D. Xia, and W.-F. Xu, "Identifying dynamic parameters of a space robot based on improved genetic algorithm," Journal of Harbin Institute of Technology, vol. 42, no. 11, pp. 1734-1739, 2010.

[10] D. Karaboga, "An idea based on honey bee swarm for numerical optimization," Tech. Rep. tr06, Computer Engineering Department, Engineering Faculty, Erciyes University, 2005.

[11] L. Ding, H. T. Wu, and Y. Yao, "Chaotic artificial bee colony algorithm for system identification of a small-scale unmanned helicopter," International Journal of Aerospace Engineering, vol. 2015, Article ID 801874, 11 pages, 2015.

[12] D. Karaboga and B. Basturk, "On the performance of artificial bee colony (ABC) algorithm," Applied Soft Computing Journal, vol. 8, no. 1, pp. 687-697, 2008.

[13] J. Swevers, W. Verdonck, and J. De Schutter, "Dynamic model identification for industrial robots," IEEE Control Systems, vol. 27, no. 5, pp. 58-71, 2007.

[14] A. Calanca, L. M. Capisani, A. Ferrara, and L. Magnani, "MIMO closed loop identification of an industrial robot," IEEE Transactions on Control Systems Technology, vol. 19, no. 5, pp. 1214-1224, 2011.

[15] N. D. Vuong and M. H. Ang Jr., "Dynamic model identification for industrial robots," Acta Polytechnica Hungarica, vol. 6, no. 5, pp. 51-68, 2009.

[16] M. Grotjahn, M. Daemi, and B. Heimann, "Friction and rigid body identification of robot dynamics," International Journal of Solids and Structures, vol. 38, no. 10-13, pp. 1889-1902, 2001.

[17] M. Gautier and W. Khalil, "A direct determination of minimum inertial parameters of robots," in Proceedings of the IEEE International Conference on Robotics and Automation., pp. 16821687, 1988.

[18] W.-X. Wu, S.-Q. Zhu, and X.-L. Jin, "Dynamic identification for robot manipulators based on modified fourier series," Journal of Zhejiang University: Engineering Science, vol. 47, no. 2, pp. 231237, 2013.
[19] C. Ganseman, J. Swevers, J. De Schutter et al., "Experimental robot identification using optimized periodic trajectories," in Proceedings of the International Conference on Noise and Vibration Engineering, pp. 585-595, 1994.

[20] J. Swevers, C. Ganseman, D. Bilgin Tükel, J. de Schutter, and H. van Brüssel, "Optimal robot excitation and identification," IEEE Transactions on Robotics and Automation, vol. 13, no. 5, pp. 730740, 1997. 

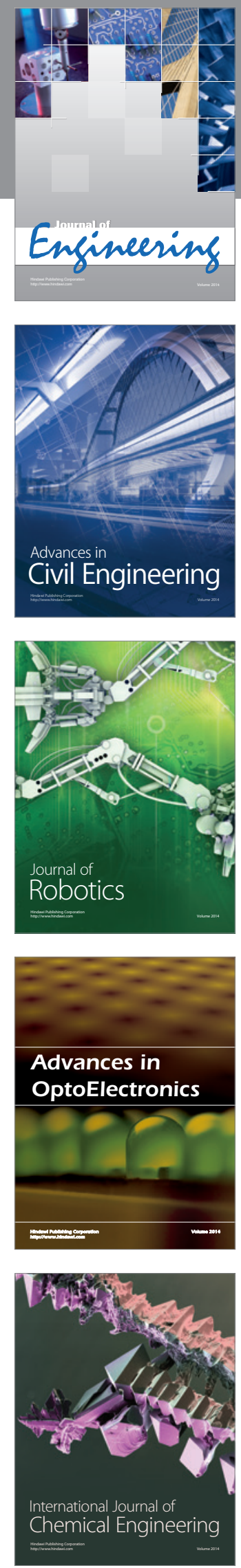

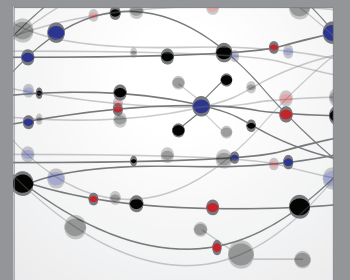

The Scientific World Journal
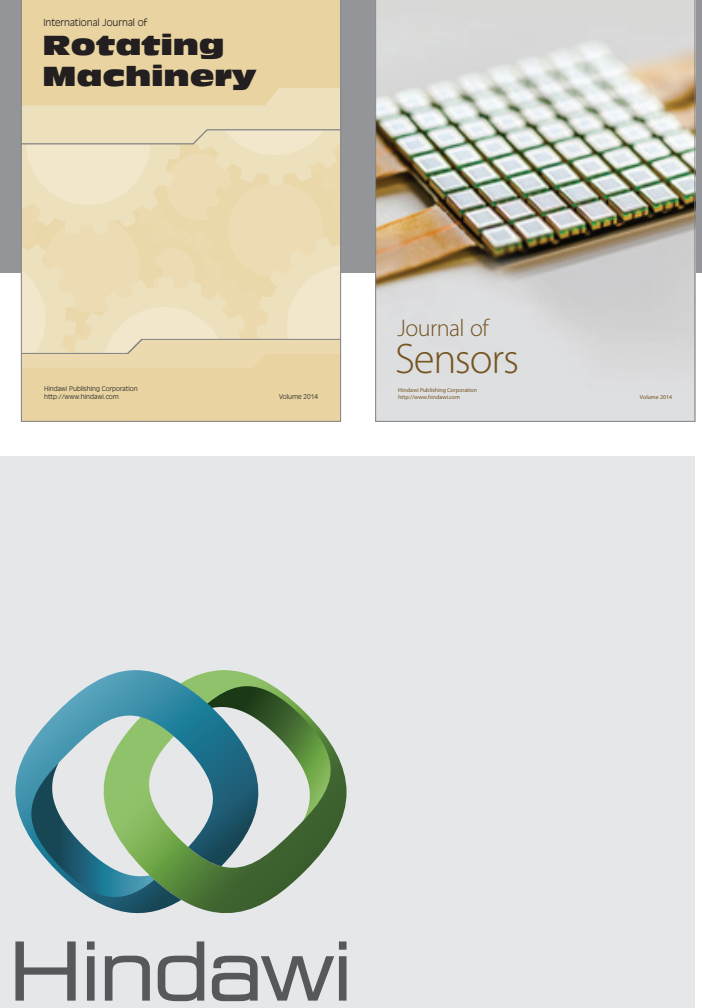

Submit your manuscripts at http://www.hindawi.com
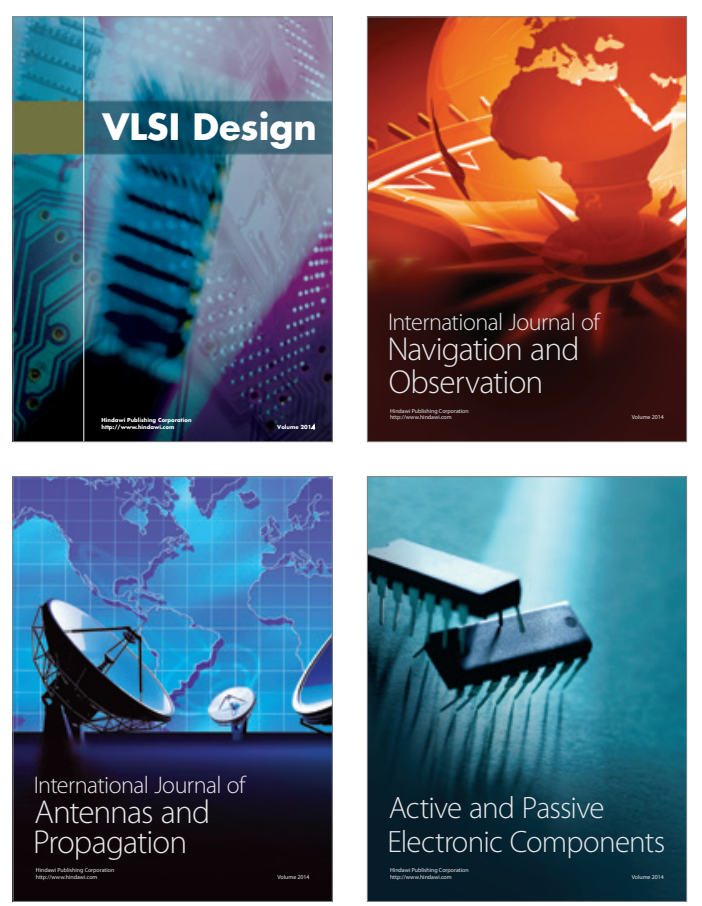
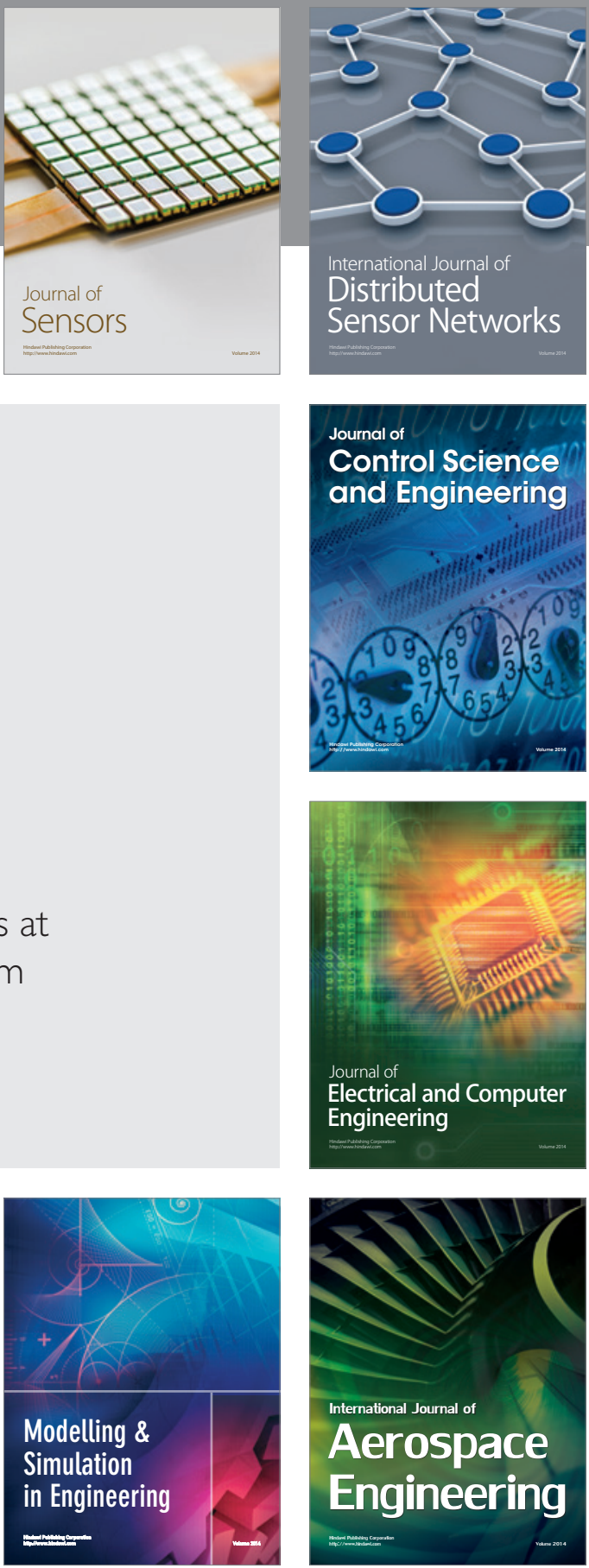

Journal of

Control Science

and Engineering
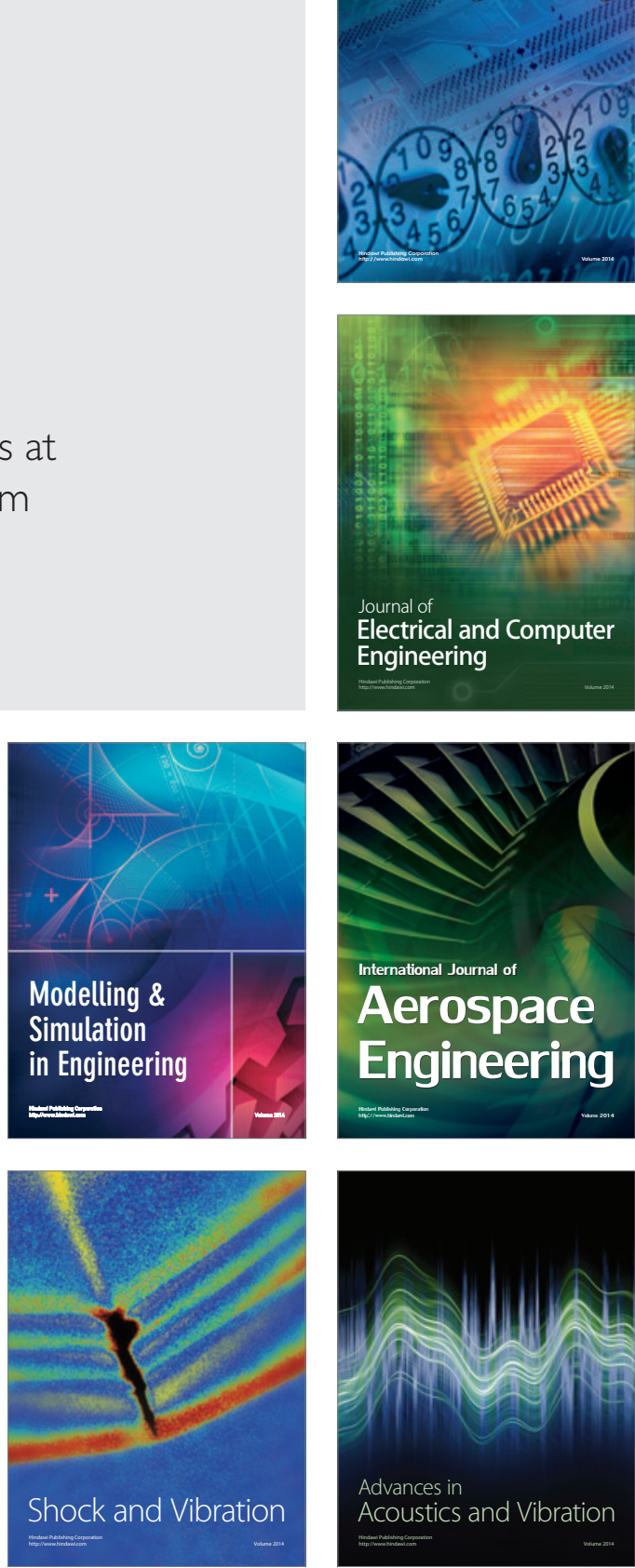